\title{
Uncertainty of the magnetic flux linkage measurements performed by modified current decay test
}

\author{
ZYGMUNT KOWALIK \\ Department of Mechatronics, Faculty of Electrical Engineering, Silesian University of Technology \\ Akademicka 2A, 44-100 Gliwice, Poland \\ e-mail: zygmunt.kowalik@polsl.pl
}

(Received: 30.09.2016, revised: 28.02.2017)

\begin{abstract}
The paper presents the estimation methodology for uncertainties of magnetic flux linkage measurements, when the flux linkage and current functions with respect to time are obtained instead of single values of these quantities. The computed uncertainties are then used to estimate the quality of an approximation of a current-flux characteristic in the mathematical model of an electrical machine when the approximation is based on the results of measurements.
\end{abstract}

Key words: flux linkage measurements, modified current decay test, uncertainty of integral calculations, uncertainty of simplicial approximation

\section{Introduction}

Mathematical models of electrical machines require the determination of the relation between currents in the windings of the machine and their flux linkages. In the models that take into account the nonlinearities of the magnetization curve the most convenient way to do it is to use an approximation of the current-flux characteristic. The current-flux characteristic is a vector function that for a given vector of phase currents assigns a corresponding vector of flux linkages. Such an approach is used in the Hamiltonian model of an electromechanical actuator [1] and it does not need calculations of any additional parameters. The opposing strategy is to determine the values of inductance matrix elements for a certain set of current values. The way of calculating data for such an approach, presented for an induction motor, but applicable in the analysis of the synchronous reluctance machines, is described in [2].

In the Hamiltonian model, the simplicial approximation of the current-flux characteristic is used. It requires sets of corresponding points in the spaces of flux linkages (space of fluxes below in the paper) and currents, and the division (triangulation) of both spaces. The sets of points can be obtained for a fixed rotor angular position using a measuring procedure that is a modification of the current decay test. However, for the measurement results to be relevant, their uncertainties have to be calculated. These calculations are also needed to determine the 
precision of the used measuring method and to designate the requirements on the measuring devices that has to be used to keep the precision of the approximation on the desired level.

In the paper, an estimation of uncertainties of the approximation of the current-flux characteristic, based on sets obtained with the use of measurement results, is presented. First, the measuring method and laboratory stand are briefly described. Then the measurement uncertainties for a single measurement of current are evaluated, followed by the estimation of uncertainties of the computed flux linkage. In the end, the impact of these uncertainties on the quality of the approximation of the flux-current characteristic is discussed.

\section{Method of measurement and laboratory stand}

The measurement of the flux linkages in the windings of the machine is performed for the fixed angular position of the rotor. For a three-phase machine with phase windings in a wye configuration, the electrical state of the machine is given by two phase current values and two corresponding generalized flux linkage values. The measuring procedure for obtaining these values consists of setting the specified current values in the machine windings, followed by their short-circuit and registration of the decaying current time plots. Corresponding flux linkage time plots are obtained by the integration of the general voltage Equation (1) that describes the machine, taking phase-to-phase voltages $e_{\mathrm{AC}}$ and $e_{\mathrm{BC}}$ equal to 0 :

$$
\frac{\mathrm{d}}{\mathrm{d} t}\left[\begin{array}{l}
\Psi_{\mathrm{AC}} \\
\Psi_{\mathrm{BC}}
\end{array}\right]=\left[\begin{array}{c}
e_{\mathrm{AC}} \\
e_{\mathrm{BC}}
\end{array}\right]-\left[\begin{array}{cc}
r_{\mathrm{A}}+r_{\mathrm{C}} & r_{\mathrm{A}} \\
r_{\mathrm{B}} & r_{\mathrm{B}}+r_{\mathrm{C}}
\end{array}\right]\left[\begin{array}{l}
i_{\mathrm{A}} \\
i_{\mathrm{B}}
\end{array}\right],
$$

where: $i_{\mathrm{A}}, i_{\mathrm{B}}$ are the currents in phase $\mathrm{A}$ and $\mathrm{B}$, respectively, $\Psi_{\mathrm{AC}}=\Psi_{\mathrm{A}}-\Psi_{\mathrm{C}}, \Psi_{\mathrm{BC}}=\Psi_{\mathrm{B}}-\Psi_{\mathrm{C}}$ are the generalized flux linkages, where $\Psi_{\mathrm{A}}, \Psi_{\mathrm{B}}, \Psi_{\mathrm{C}}$ are the flux linkages in winding of phase $\mathrm{A}, \mathrm{B}$ and $\mathrm{C}$, respectively, $r_{\mathrm{A}}, r_{\mathrm{B}}, r_{\mathrm{C}}$ are the resistances of windings connected to phase $\mathrm{A}, \mathrm{B}$ and $\mathrm{C}$, respectively.

The result of a single measurement done with the use of this method is a pair of corresponding trajectories, one in the space of fluxes and the second in the space of currents. From this pair of trajectories, several points, best characterizing the curvature, can be chosen to be included into the required sets. The selection of points is based on the magnetic field coenergy calculations. The method of measurement and selection of points included into the required sets is described in literature [3].

The presented measurement results are obtained for a prototype three phase Synchronous Reluctance Machine with wye-connected phase windings, with the rotor in an arbitrary position (neither aligned nor unaligned). This prototype machine has 4 poles and is built based on the stator of an induction machine, with rated parameters $I_{\mathrm{N}}=2.2 \mathrm{~A} ; P_{\mathrm{N}}=0.55 \mathrm{~kW}$; and with 36 winding slots. In such a machine the magnetic saturation effects are well visible during normal work and hence the Hamilton approach is well suited to describe it. However, the results should be treated as an illustration of the presented method of uncertainties calculation rather than the construction of the precise model of this particular machine. The laboratory stand that was used during measurements is presented in Fig. 1 and is described in details in literature [4]. 
Synchronous Reluctance Machine

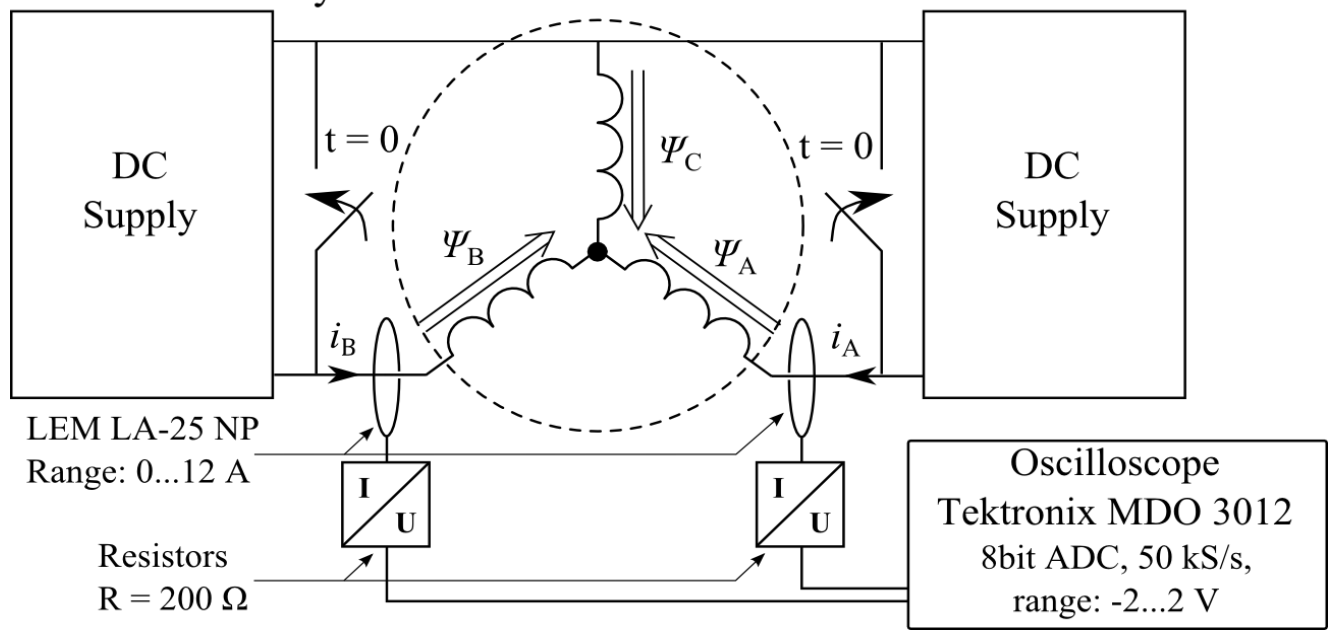

Fig. 1. Schematic view of the used laboratory stand

During a single measurement, for a fixed DC supply, the decaying time plots are sampled and registered 4 times, and then they are averaged to increase the precision of measurement and reduce the random noise influence. At this stage, the random (Type A uncertainties) and the systematic (Type B uncertainties) effects can be assessed. The acquired and averaged time plots in general contain a non-decaying constant component, which is caused e.g. by unbalanced amplifiers that are used in the measuring devices. This constant can be eliminated by finding the time value $T_{0}$, after which the "real", decaying time plots of currents go to 0 . It can be done by the analysis of the magnetic coenergy time plot $E_{\mathrm{cm}}(t)$ that is computed with the use of obtained time plots of currents (2):

$$
E_{\mathrm{cm}}(t)=\int_{t}^{T}\left(\Psi_{\mathrm{AC}}(\tau) \frac{\mathrm{d} i_{\mathrm{A}}(\tau)}{\mathrm{d} \tau}+\Psi_{\mathrm{BC}}(\tau) \frac{\mathrm{d} i_{\mathrm{B}}(\tau)}{\mathrm{d} \tau}\right) \mathrm{d} \tau,
$$

where time plots $i_{\mathrm{A}}(\tau), i_{\mathrm{B}}(\tau), \Psi_{\mathrm{AC}}(\tau), \Psi_{\mathrm{BC}}(\tau)$ are registered in the time range from 0 to $T$. After the coenergy computation, time $T_{0}$ is found to be the time that ensures $E_{\mathrm{cm}}\left(T_{0}\right) \approx 0$. The obtained time plots of currents are then "cut" to this moment and the mean value on the interval $\left(T_{0}, T\right)$ is computed. This value is the constant component that needs to be filtered (subtracted) from the measurement results. It has to be clearly stated that the above procedure is a part of preprocessing of the measured time plots of currents, as the correct time plots of flux linkages and coenergy can be computed only after getting rid of the non-decaying component from the obtained time plots of currents. The processing of the measurement results is shown for exemplary time plots of currents in Fig. 2 and Fig. 3.

Fig. 2 presents the time plot obtained using a LEM transducer, that measures the current in the windings of phase A (the time plot obtained with the use of LEM that measures the current in the B phase is similar). Both full time plot and the decaying fragments are denoted. Fig. 3 presents the decaying time plots of currents computed using fragments shown in Fig. 2, the 
decaying time plot of coenergy used in measurements processing and finally the decaying time plots of fluxes computed using the processed time plots of currents. The time value $T_{0}$ is marked in the figure.

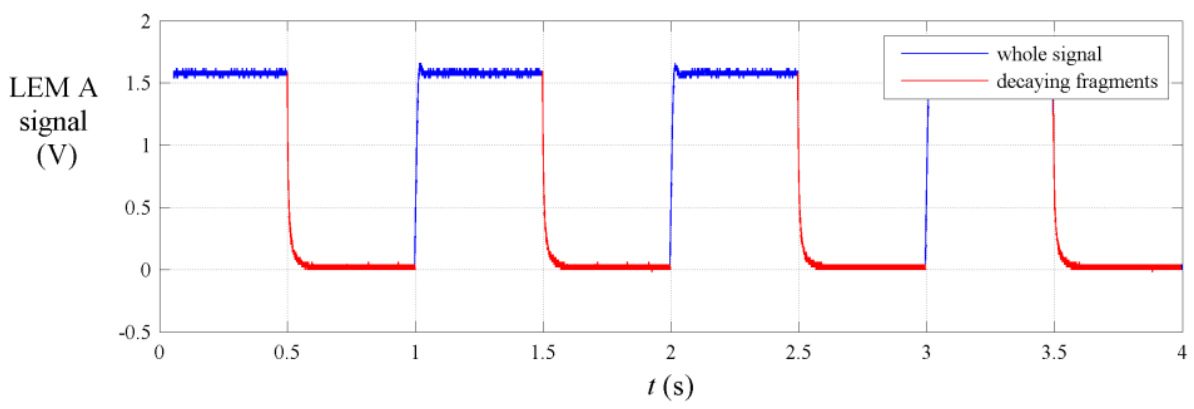

Fig. 2. Time plot registered during the measuring procedure
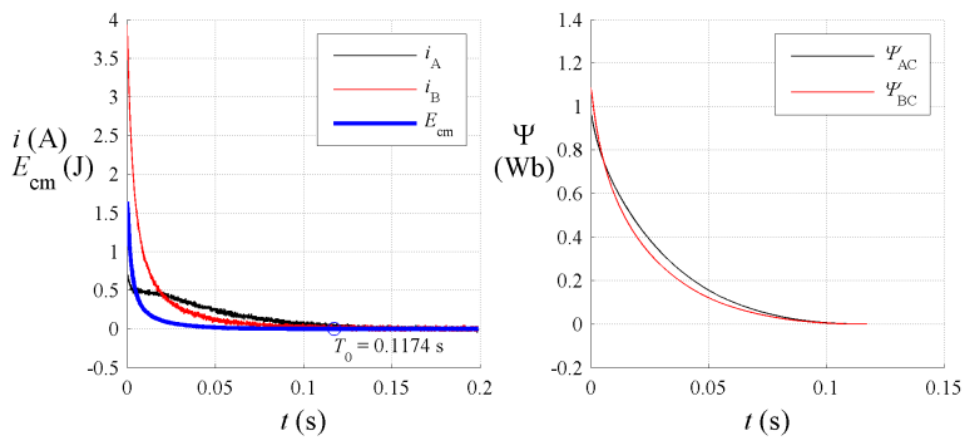

Fig. 3. Decaying time plots of currents, coenergy (left) and computed generalized flux linkages

\section{Uncertainties of current and flux linkages measurements}

In the calculation of uncertainties of current measurements, standard procedures of determining the Type A and Type B uncertainties were used to obtain the uncertainty of every sample of the measured time plots of currents. The Type A uncertainty, denoted $u_{\mathrm{A}}$, was computed as a square root of the variation, further extended by multiplying it by the fractile $t_{3, \alpha}=1.20$ of the student distribution for a confidence level $\alpha=0.6827$. The Type B uncertainty, denoted $u_{\mathrm{B}}$, was computed using technical documentations of the used measuring equipment and took into account the resolution of the used analog-to-digital converter (ADC), the accuracy of the DC gain of the used oscilloscope, the accuracy and linearity error of the used LEM transducers and uncertainty of the resistances connected to their outputs and used as the current to voltage transducers. 
The standard uncertainty of the measurements of currents affects the uncertainty of the calculated flux linkages. The process of determining this uncertainty will be shown for a $\Psi_{\mathrm{AC}}$ flux linkage and is analogous for a $\Psi_{\mathrm{BC}}$ flux. It starts from the formula for computing the values of the generalized flux $\Psi_{\mathrm{AC}}(t)$ in a given moment in time $t$, based on (1), which is as follows:

$$
\Psi_{\mathrm{AC}}(t)=-\left(r_{\mathrm{A}}+r_{\mathrm{C}}\right) \int_{t}^{T_{0}} i_{\mathrm{A}}(\tau) \mathrm{d} \tau-r_{\mathrm{A}} \int_{t}^{T_{0}} i_{\mathrm{B}}(\tau) \mathrm{d} \tau .
$$

The law of uncertainty propagation applied to (3) yields the square of standard uncertainty $u\left(\Psi_{\mathrm{AC}}(t)\right)$ to be equal to:

$$
\begin{aligned}
u^{2}\left(\Psi_{\mathrm{AC}}(t)\right)= & {\left[u^{2}\left(r_{\mathrm{A}}\right)+u^{2}\left(r_{\mathrm{C}}\right)\right] I_{i \mathrm{~A}}^{2}(t)+u^{2}\left(r_{\mathrm{A}}\right) I_{i \mathrm{~B}}^{2}(t)+} \\
& +\left(r_{\mathrm{A}}+r_{\mathrm{C}}\right) u^{2}\left(I_{i \mathrm{~A}}(t)\right)+r_{\mathrm{A}} u^{2}\left(I_{i \mathrm{~B}}(t)\right)
\end{aligned},
$$

where: $I_{i \mathrm{~A}}(t), I_{i \mathrm{~B}}(t)$ are the integrals of the time plot of the respective current from $t$ to $T_{0}$. Because the measured data are discrete time series, the above integrals are approximated with sums:

$$
\boldsymbol{I}_{i \mathrm{~A}}(n)=\sum_{j=n}^{N} \boldsymbol{i}_{\mathrm{A}}(j) \Delta t, \quad \boldsymbol{I}_{i \mathrm{~B}}(n)=\sum_{j=n}^{N} \boldsymbol{i}_{\mathrm{B}}(j) \Delta t,
$$

where: $\Delta t$ is the time between subsequent current samples, $N$ is the number of samples in the whole discrete time plot of current, $\boldsymbol{i}_{\mathrm{A}}, \boldsymbol{i}_{\mathrm{B}}, \boldsymbol{I}_{i \mathrm{~A}}$ and $\boldsymbol{I}_{\mathrm{iB}}$ are the column vectors that replace the continuous functions $i_{\mathrm{A}}(t), i_{\mathrm{B}}(t), I_{i \mathrm{~A}}(t)$ and $I_{i \mathrm{~B}}(t)$ registered in time interval $t \in\left\langle 0, T_{0}\right\rangle$, consisting of samples of the respective time plots. All the calculations are in fact performed on these vectors.

To compute the standard uncertainties of those sums when the time series of Type A and Type B uncertainties are given separately, the approach described in [5] can be used. Using it the uncertainties of the integrals $u\left(I_{i \mathrm{~A}}(t)\right), u\left(I_{i \mathrm{~B}}(t)\right)$ are approximated by their discrete analogues, e.g. computation of $u\left(I_{i \mathrm{~A}}(t)\right)$ leads to the formula:

$$
u^{2}\left(I_{i \mathrm{~A}}(t)\right) \approx u^{2}\left(\boldsymbol{I}_{i \mathrm{~A}}(n)\right)=\sum_{j=n}^{N}\left[\Delta t \cdot u_{\mathrm{A}}\left(\boldsymbol{i}_{\mathrm{A}}(j)\right)\right]^{2}+\left[\Delta t \sum_{j=n}^{N} u_{\mathrm{B}}\left(i_{\mathrm{A}}(j)\right)\right]^{2},
$$

where: $u\left(\boldsymbol{I}_{i \mathrm{~A}}(n)\right)$ is the uncertainty of $n$-th sample in the integral of the $\boldsymbol{i}_{\mathrm{A}}$ current time series, with the $n$-th sample corresponding to the $t$ moment in time, $u_{\mathrm{A}}\left(\boldsymbol{i}_{\mathrm{A}}(j)\right), u_{\mathrm{B}}\left(\boldsymbol{i}_{\mathrm{A}}(j)\right)$ are the Type A and B uncertainty of the $j$-th sample in the $\boldsymbol{i}_{\mathrm{A}}$ current time series. Formulas (5) and (6) are valid for a constant sampling rate and for the computation of integrals using the rectangle method.

In the Equation (6) the law of propagation is strictly applied only to the random part of uncertainty, i.e. the integral of the random uncertainties is treated as a simple sum. That way the random uncertainties are greatly diminished, whereas the systematic errors "pass" through 
the calculations. It is consistent with intuition - integration filters the random noise, but when the integrated data differ from the true values "by a percentage", the result of calculations should also differ from the true result by the same percentage.

The average relative uncertainty of flux linkages values, computed using Formulas (4), (5) and (6) is equal to $20.93 \%$, and the maximum value of this uncertainty is equal to $32.28 \%$, which is very pessimistic considering the average relative uncertainty of the current equal to $4.87 \%$. To check these calculations, another approach was used to obtain the time plots of flux linkages. This approach and Formulas (7)-(9) are introduced in [6]. Similarly to the earlier approach, here only the equations for computing the uncertainty of $\boldsymbol{I}_{i \mathrm{~A}}$ integral are presented, as the calculations for computing the $\boldsymbol{I}_{i \mathrm{~B}}$ integral are analogical. In this approach the time plots of currents are approximated using Chebyshev polynomials of the first kind. The column vector $\boldsymbol{\alpha}$ consisting of $m_{C h}$ coefficients is computed so that the error $\varepsilon$, written for the $N \mathrm{H} 1$ column vector $\boldsymbol{i}_{\mathrm{A}}$, is minimized:

$$
\varepsilon^{2}=\left\|\boldsymbol{C} \boldsymbol{\alpha}-i_{\mathrm{A}}\right\|^{2}=\left(\boldsymbol{C} \boldsymbol{\alpha}-i_{\mathrm{A}}\right)^{T}\left(\boldsymbol{C} \boldsymbol{\alpha}-i_{\mathrm{A}}\right),
$$

where $\boldsymbol{C}$ is the $N \mathrm{H} m_{C h}$ matrix, where column $k$ stores samples of the Chebyshev polynomial of degree $k-1$. The minimization of the error in (7) leads to the coefficients in $\boldsymbol{\alpha}$ being a linear combination of the samples in the $\boldsymbol{i}_{\mathrm{A}}$ vector:

$$
\boldsymbol{\alpha}=\left(\boldsymbol{C}^{T} \boldsymbol{C}\right)^{-1} \boldsymbol{C}^{T} \boldsymbol{i}_{\mathrm{A}}=\boldsymbol{M} \boldsymbol{i}_{\mathrm{A}} .
$$

Similarly, the integral of the approximated time plot of currents is a linear combination of the integrated Chebyshev polynomials, which can be computed analytically. When the column vectors of samples of these integrals build a matrix $\boldsymbol{I}_{\boldsymbol{C}}$ similar to the matrix $\boldsymbol{C}$, the integral column vector $\boldsymbol{I}_{i \mathrm{~A}}$ can be evaluated as:

$$
I_{i \mathrm{~A}}=I_{C} \boldsymbol{\alpha}=I_{C} M i_{\mathrm{A}}=N i_{\mathrm{A}} .
$$

The true values of elements of the matrices $\boldsymbol{I}_{\boldsymbol{C}}$ and $\boldsymbol{M}$ are known, and thus the computation of an integral is simply a weighted sum. Applying the law of uncertainty propagation to (9) results in the uncertainties of subsequent samples in the $\boldsymbol{I}_{i \mathrm{~A}}$ vector that fulfills:

$$
u^{2}\left(\boldsymbol{I}_{i \mathrm{~A}}(n)\right)=\sum_{j=1}^{N} \boldsymbol{N}^{2}(n, j) \cdot u^{2}\left(\boldsymbol{i}_{\mathrm{A}}(j)\right),
$$

where $N^{2}(n, j)$ is the squared value from $n$-th row and $j$-th column of the matrix $N$, $u^{2}\left(\boldsymbol{i}_{\mathrm{A}}(j)\right)=u_{\mathrm{A}}^{2}\left(\boldsymbol{i}_{\mathrm{A}}(j)\right)+u_{\mathrm{B}}^{2}\left(\boldsymbol{i}_{\mathrm{A}}(j)\right)$ is the square of the standard uncertainty of the $j$-th sample in the $\boldsymbol{i}_{\mathrm{A}}$ vector.

The relative uncertainties of flux linkages computed using the Chebyshev approximation are much more optimistic, being equal to $2.98 \%$ in average, with a maximum value equal to $4.51 \%$. At the same time, the results of flux linkages calculations using the $m_{C h}=14$ Chebyshev polynomials to approximate time plots of currents were very close to the results obtained using a simple rectangle method. 
Using two different methods of calculations on the same dataset and obtaining the same results should also result in similar values of uncertainties "connected" to both methods. This leads to the hypothesis that the methods of uncertainties computation require additional information to make the results consistent.

As this information, the fact that the acquired time plots of currents have to decay to 0 can be used. With such an assumption, even if at the start of the measuring procedure the initial values of the currents were measured with a significant error, both measured and "real" trajectories (in both space of currents and space of fluxes) have to come closer to each other as the system evolves, as long as the system is not chaotic. Considering uncertainties as a measure of the distance between "real" and measured trajectories, it can be assumed that the uncertainties also become smaller and decay at the rate similar to the rate of decay of the time plots of currents. In other words the influence of the uncertainty is the greatest at the start of the measurement (when the initial point of the trajectory to be measured is fixed) and diminishes as the system evolves to the known point $(0,0)$.

The decay of the measured time plots is approximately exponential. For a given vector of samples, e.g. $\boldsymbol{i}_{\mathrm{A}}$, the biggest positive parameter $\lambda$ can be found that satisfies the formula:

$$
\forall n \leq N:\left(i_{p 1}-i_{p 2}\right) e^{-\lambda n \Delta t}+i_{p 2}>\left|\boldsymbol{i}_{\mathrm{A}}(n)\right|,
$$

where: $i_{p 1}, i_{p 2}$ are the positive parameters chosen at the start of the procedure of finding $\lambda$. The parameter $i_{p 1}$ was set as $\max \left(\left|i_{\mathrm{A}}\right|\right)$ assuming that in most cases the biggest value of the acquired time plot of a current is measured at the start of the time plot, and the parameter $i_{p 2}$ was set to be the maximum absolute value of the "tail" of the acquired time plot (in most cases $i_{p 2}$ was small), so that the expression at the left side of the inequality in (11) does not decay to 0 as $n \rightarrow N$. In practical calculations it was also permitted to violate (11) for a certain low number of $\boldsymbol{i}_{\mathrm{A}}(n)$ values to make the process of finding the biggest possible $\lambda$ more robust.

After the parameter $\lambda$ is found, the new, bounded random uncertainty of the current in phase $\mathrm{A} u_{\mathrm{A} b}\left(\boldsymbol{i}_{\mathrm{A}}\right)$ are evaluated.

$$
u_{\mathrm{A} b}\left(\boldsymbol{i}_{\mathrm{A}}\right)=\left(\frac{i_{p 1}-i_{p 2}}{i_{p 1}} e^{-\lambda n \Delta t}+\frac{i_{p 2}}{i_{p 1}}\right) u_{\mathrm{A}}\left(\boldsymbol{i}_{\mathrm{A}}\right) .
$$

Similar calculations are performed for systematic uncertainty $u_{\mathrm{B} b}\left(\boldsymbol{i}_{\mathrm{A}}\right)$. In these computations, the uncertainties obtained during measurements are multiplied by a "normalized" time series of the left side of the inequality (11) that decays from 1 to $i_{p 1} / i_{p 2}$. Then, those new values of uncertainties are incorporated into the previously introduced methods ((6), (10) and then (4)).

The results of computations for an exemplary time plot of current $i_{\mathrm{A}}$ is shown in Figure 4. A time plot $f(t)$ of type given by the (11), that decays at the rate similar to the time plot of current, is shown on the left, and the standard uncertainties of the considered time plot of currents, both unbounded $u\left(i_{\mathrm{A}}\right)$ and bounded $u_{b}\left(i_{\mathrm{A}}\right)$, are shown on the right.

The average and maximum values of the relative uncertainties, computed using different methods, are shown in Table 1.

The results of computations is satisfactory and consistent, although they may seem to be too optimistic. It should be also pointed out, that although bounding the uncertainties of 
measured currents is useful in flux linkages calculations, this uncertainty should not be applied when measuring the arbitrary values of currents.
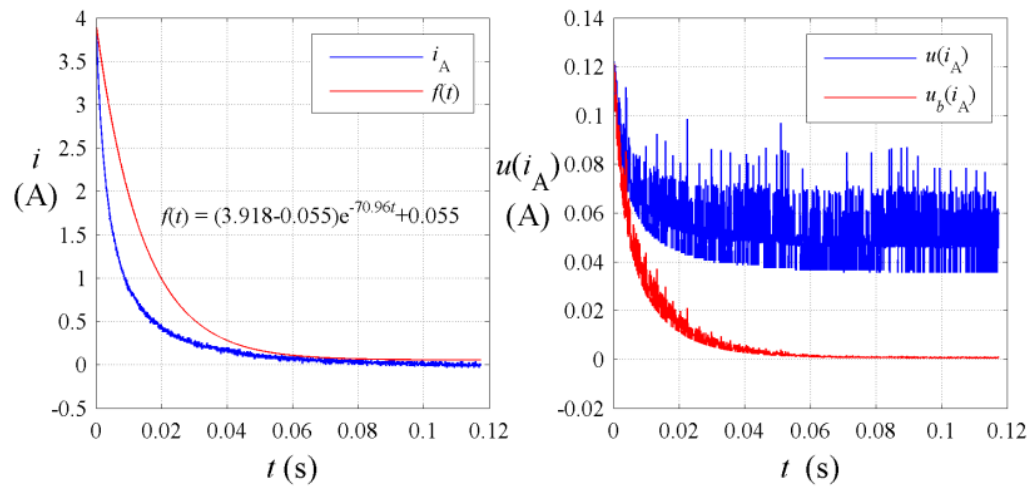

Fig. 4. An exemplary time plot of current against the selected time plot $f(t)$ (left) and its bounded and unbounded standard uncertainty

Table 1. Relative uncertainties of currents and flux linkages, computed using different methods. "Bounded" values refer to (11) and (12)

\begin{tabular}{l|c|c|c|c|c|c}
\hline \multirow{2}{*}{$\begin{array}{c}\text { Relative } \\
\text { uncertainty, } \\
\boldsymbol{\%}\end{array}$} & \multicolumn{2}{|c|}{ Current measurements } & \multicolumn{4}{c}{ Flux linkages computations } \\
\cline { 4 - 7 } & unbounded & \multirow{2}{*}{ bounded } & \multicolumn{2}{c|}{ using rectangle method } & \multicolumn{2}{c}{ using Chebyshev appr. } \\
\cline { 4 - 7 } & & & unbounded & bounded & unbounded & bounded \\
\hline average & 4.87 & 3.72 & 20.93 & 4.50 & 2.98 & 2.35 \\
\hline maximum & 10.72 & 6.64 & 32.28 & 14.21 & 4.51 & 3.82 \\
\hline
\end{tabular}

The reason for this is that the bounding procedure is suited only for decaying current time plots. In other words, when measuring values of currents in a certain point of the space of currents, uncertainties determine the rectangle where the "real" point should be found - this rectangle "lessens" its size only along the decaying trajectory that starts in the initially measured point. If one would like to later measure the values of currents in a point that is located on this trajectory, the uncertainties of this measurement are bigger than the bounded ones, e.g. due to the uncertainty of the way of forcing this desired current values in the windings of an examined electrical machine.

After the analysis of Table 1 and considering the computational effort connected to the examined methods, a method of flux linkages computation using the rectangle method of integration and bounded uncertainties of currents along the integrated time plots of currents is chosen as the best.

\section{The approximation of the current-flux characteristic}

As stated earlier, the goal of the measurements is to build sets of corresponding points located in the spaces of currents and fluxes. These points are chosen from the measured and 
computed trajectories, along with their corresponding uncertainties. The chosen points with error bars, the representative pair of corresponding trajectories in both spaces and the areas of these spaces determined by the calculated uncertainties are presented in Fig. 5.
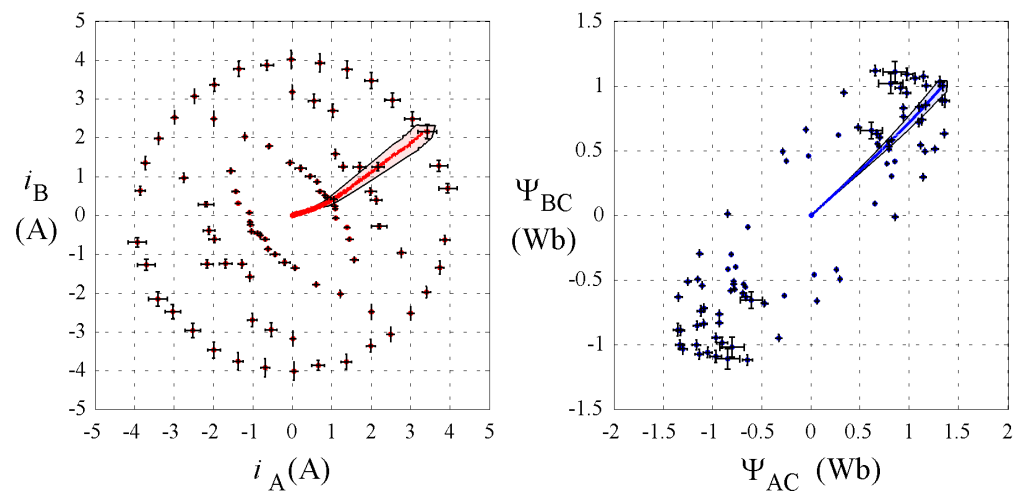

Fig. 5. Sets of points chosen to approximate the flux-current characteristic of the analyzed electrical machine

The approximation of the current-flux characteristic performed with the use of simplicial approximation is of course affected by these uncertainties. To compute this influence, the process of approximating the characteristic should be briefly introduced. It starts with the measurement of the values of currents in phases A and B in a single moment in time, that determine a single point $\boldsymbol{i}_{p}=\left(i_{\mathrm{A} p} ; i_{\mathrm{B} p}\right)$ in the space of currents. The standard uncertainty of the measured value is known to be $u\left(\boldsymbol{i}_{p}\right)$. To find the corresponding point $\boldsymbol{\Psi}_{p}$ in the space of fluxes, first a simplex (a triangle-shaped subarea of the space of currents, determined by three points $\left.\boldsymbol{i}_{k}, \boldsymbol{i}_{l}, \boldsymbol{i}_{m}\right)$ that contains the point $\boldsymbol{i}_{p}$ has to be found. Then the barycentric coordinates $\boldsymbol{b}=\left[b_{k} b_{l} b_{m}\right]^{T}$ that determine the position of the point $\boldsymbol{i}_{p}$ in the found simplex are calculated, fulfilling:

$$
\left[\begin{array}{ccc}
i_{\mathrm{A} k} & i_{\mathrm{A} l} & i_{\mathrm{A} m} \\
i_{\mathrm{B} k} & i_{\mathrm{B} l} & i_{\mathrm{B} m} \\
1 & 1 & 1
\end{array}\right]\left[\begin{array}{c}
b_{k} \\
b_{l} \\
b_{m}
\end{array}\right]=\left[\begin{array}{c}
i_{\mathrm{A} p} \\
i_{\mathrm{B} p} \\
1
\end{array}\right],
$$

where e.g. $\boldsymbol{i}_{k}=\left(i_{\mathrm{A} k} ; i_{\mathrm{B} k}\right)$ and so on. Because the points $\boldsymbol{i}_{k}, \boldsymbol{i}_{l}, \boldsymbol{i}_{m}$ have their corresponding points $\boldsymbol{\Psi}_{k}, \boldsymbol{\Psi}_{l}, \boldsymbol{\Psi}_{m}$ in the space of fluxes, the point $\boldsymbol{\Psi}_{p}$ lies in the simplex determined by them.

$$
\left[\begin{array}{l}
\Psi_{\mathrm{AC} p} \\
\Psi_{\mathrm{BC} p}
\end{array}\right]=\left[\begin{array}{lll}
\Psi_{\mathrm{AC} k} & \Psi_{\mathrm{ACl}} & \Psi_{\mathrm{AC} m} \\
\Psi_{\mathrm{BC} k} & \Psi_{\mathrm{BC} l} & \Psi_{\mathrm{BC} m}
\end{array}\right]\left[\begin{array}{c}
b_{k} \\
b_{l} \\
b_{m}
\end{array}\right],
$$

where e.g. $\Psi_{k}=\left(\Psi_{\mathrm{AC} k} ; \Psi_{\mathrm{BC} k}\right)$ and so on. Using the law of propagation to (14) to calculate the standard uncertainty $u\left(\Psi_{\mathrm{AC} p}\right)$ yields: 
$u^{2}\left(\Psi_{\mathrm{AC} p}\right)=\left[\begin{array}{lll}u^{2}\left(\Psi_{\mathrm{AC} k}\right) & u^{2}\left(\Psi_{\mathrm{ACl}}\right) & u^{2}\left(\Psi_{\mathrm{AC} m}\right)\end{array}\right]\left[\begin{array}{c}b_{k}{ }^{2} \\ b_{l}{ }^{2} \\ b_{m}{ }^{2}\end{array}\right]+\left[\begin{array}{lll}\Psi_{\mathrm{AC} k} & \Psi_{\mathrm{ACl}} & \Psi_{\mathrm{AC} m}\end{array}\right] \Sigma(\boldsymbol{b})\left[\begin{array}{c}\Psi_{\mathrm{AC} k} \\ \Psi_{\mathrm{ACl}} \\ \Psi_{\mathrm{AC} m}\end{array}\right]$

and similarly to calculate the standard uncertainty $u\left(\Psi_{\mathrm{BC} p}\right)$. In (15) $\Sigma(\boldsymbol{b})$ is a covariance matrix of the vector $\boldsymbol{b}$ containing barycentric coordinates. Calculation of the values of its elements requires the calculation of the covariance matrix of the inverse of the $3 \mathrm{H} 3$ matrix that can be found in (13). The method for computing the covariance matrix that was used in the paper is described in [7].

To test an approximation based on the sets of points shown in Fig. 5, an approximation of the points located in the middle of every simplex (i.e. every barycentric coordinate equal to $1 / 3$ ) from the triangulated set of points in the space of currents was performed. Then the uncertainty of the approximated flux linkages value was computed using (15), taking the uncertainty of the current value equal to $u\left(i_{p}\right)=\left(u\left(i_{\mathrm{Asim}}\right)+0.05 ; u\left(i_{\mathrm{B} s i m}\right)+0.05\right)(\mathrm{A})$, where $u\left(i_{\mathrm{Asim}}\right)=\left(u\left(i_{\mathrm{A} k}\right)+u\left(i_{\mathrm{Al}}\right)+u\left(i_{\mathrm{A} m}\right)\right) / 3$ is the average uncertainty of the "phase $\mathrm{A}$ " coordinate of the points in the vertices of a considered simplex $\left(u\left(i_{\mathrm{Bsim}}\right)\right.$ is computed similarly). The results of computations for determining a relative uncertainty of the $\Psi_{\mathrm{AC} p}$ value are shown in Fig. 6.

The average uncertainty of the $\Psi_{\mathrm{AC} p}$ value is equal to $6.54 \%$, while the maximum value of this uncertainty was determined to be equal to $13.74 \%$. In Fig. 6 it can be seen that the computed uncertainties are bigger for the points, that in the space of currents are located near the line $i_{\mathrm{A}}=-i_{\mathrm{B}}$, where the flux-current characteristic is known to be highly nonlinear. In the regions where both $i_{\mathrm{A}}$ and $i_{\mathrm{B}}$ components are big in absolute value and have the same sign, the computed uncertainty is at the rate of $4-5 \%$.

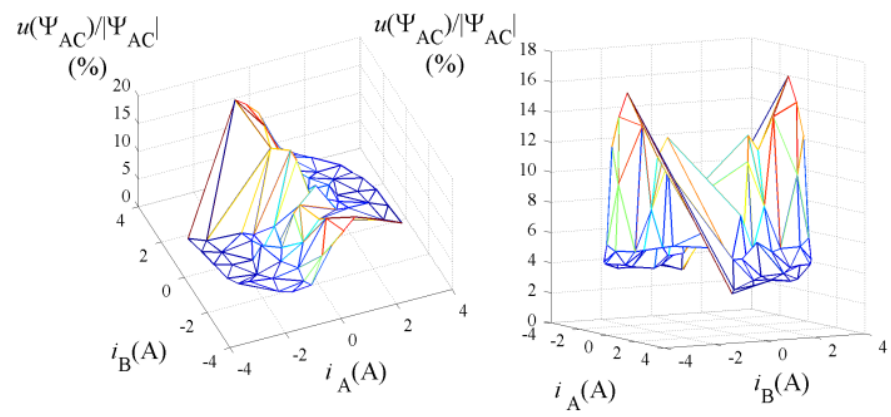

Fig. 6. Relative uncertainty of the approximated $\Psi_{\mathrm{AC}}$ flux value viewed from two angles

\section{Conclusions}

In the paper, a methodology for evaluating the uncertainty of the simplicial approximation of the flux-current characteristic based on data obtained during measurements is shown. 
First, the method of computing the uncertainty of the modified current decay test is presented. Because flux linkages are coupled with the measured decaying time plots of currents by an integral equation, the main problem was to determine the uncertainty related to the computed integrals of these time plots. The final uncertainty value can be significantly lowered with the use of the information that the time plots of a current have to decay to 0 and using the computed coenergy value to find the time of this decay.

From the computed decaying time plots of currents and flux linkages, several points can be chosen to the sets that the simplicial approximation of the flux-current characteristic is based on. Those points have their associated uncertainty that propagates on the approximated flux linkage value when using the aforementioned sets of points. The method of computing this uncertainty is also presented.

The presented methods were used to evaluate the uncertainty of the approximation of the flux-current characteristic for an exemplary synchronous reluctance machine. The relative uncertainty of the approximation can be evaluated to be approximately $7 \%$, which is sufficient for the use in the Hamiltonian model of the examined machine. It is worth noting that the measurements leading to this result were performed using relatively low requirements on the data acquisition equipment. The presented methods of measurements and approximation can thus be used in the control system of an electrical machine (at least considering the measurement part of it) without high requirements concerning the used measurement devices.

\section{References}

[1] Burlikowski W., Hamiltonian model of electromechanical actuator in natural reference frame, Part 1\&2, Archives of Electrical Engineering, vol. 60, no. 3, pp. 317-348 (2011).

[2] Demenko A., Nowak L., Pietrowski W., Calculation of magnetization characteristic of a squirrel cage machine using edge element method, COMPEL-The international journal for computation and mathematics in electrical and electronic engineering, vol. 23, issue 4, pp. 1110-1118 (2004).

[3] Kowalik Z., Measurement results based approximation of the flux-current characteristic of an electrical actuator, Measurement, Automation, Monitoring, vol. 62, no. 02, pp. 49-54 (2016).

.[4] Burlikowski W., Kohlbrenner Ł., The measurement test for the identification of current-flux characteristic in synchronous reluctance motors, Measurement, Automation, Monitoring, vol. 62, no. 01, pp. 33-36 (2016).

[5] Wooliams E.R., Determining the uncertainties associated with integrals of spectral quantities, A report of EMRP Joint Research EMRP-ENG05-1.3.1 (2013).

[6] Vance R., A note on error propagation with an integral, http://physics.stackexchange.com/ questions/196957/error-propagation-with-an-integral, accessed 20 Jul 2016.

[7] Lefebvre M., Keeler R.K., Sobie R., White J., Propagation of errors for matrix inversion, Nucl. Instrum. Meth., no. A451, pp. 520-528 (2000). 